\title{
Investigation on rotating compression of porous cylinder under constant shear friction using FEM simulation
}

\author{
Gow-Yi Tzou ${ }^{1}$, Yeong-Maw Hwang ${ }^{2}$, and Sai-Chih Pan $^{2, *}$ \\ ${ }^{1}$ Department of Mechanical and Automation Engineering, Chung Chou University of Science and \\ Technology, Yuanlin, Changhua County 510, Taiwan, R.O.C. \\ ${ }^{2}$ Department of Mechanical and Electro-Mechanical Engineering, National Sun Yat-Sen University, \\ Kaohsiung 804, Taiwan, R.O.C.
}

\begin{abstract}
In this paper, a series of systematic studies on the rotating compression of porous cylinders by the FEM using DEFORM-3D analysis software are carried out. For a series of simulations, the forming conditions such as (1) the angular velocities from small to large are $0,0.1,0.25,0.5$, and $1(\mathrm{rad} / \mathrm{sec})$, respectively;(2) aspect ratio of porous cylinder (heightdiameter ratio) are $0.75,1$, and 1.25 , respectively;(3) the frictional factors are 0.3 and 0.7 . Through different combinations of the above-mentioned variation factors, the characteristics of the rotating compression of porous cylinder can be explored. These rotating forming characteristics such as the compression force, the bulging ratio, the change in density, the rotating torque and so on can be investigated. The rotating compression characteristics of both porous and sound cylinders reveal lots of similarities and some differences. The main conclusions of this study are as follows: (1) as the rotating speed increases fast, the compression force of porous cylinder is whereas reduced. This phenomenon is different from the compression of sound cylinder; (2)the compression force under the lower aspect ratio can be reduced by the lubrication; (3)the relative density can be increased by increasing the rotating speed and reduction ratio; (4)the rotating torque is increased with higher rotating speed, higher frictional factor, and higher aspect ratio; (5)the bulging effect can be reduced by increasing the rotating speed, however it is increased by increasing the frictional factor.
\end{abstract}

\section{Introduction}

Porous materials can be manufactured by the process of casting or powder metallurgy. For the contracted volume of metal slabs or the constrained air within the metal slabs during the casting, there are tiny voids appeared; especially on or within the thick part easy to generate the voids. It is hard to observe the existed voids without a microscope. These tiny voids will affect the pressure resistance and strength of the products. For the products manufactured by the power metallurgy, if the technology of powder forging technology can

* Corresponding author:scpan@seed.net.tw 
be used, more functional and good mechanical properties as well as the alloys in different compositions will be acquired.

In the paper of Yang et al. [1] explored the porous materials on the relationship of bulging deformation, forging force and density change during open die forging. By FEM analysis and other experiment results, they investigated the applicability of FEM simulations. Lee et al. [2] emphasized the importance of the empty hole effect and the density distribution in the study of porous materials. In addition to their guideline on plastic yield, they also compared with other guidelines on yield in various studies. Using the stress-strain curve data in the sintered copper experiment, Shima et al. [3] proposed the plasticity theory for porous metals and applied it to a friction-free closed-dies compression experiment to verify its practicability. Doraivelu et al. [4] reviewed many yield criteria proposed by various scholars. They detailed the compressible powder metallurgy materials, proposed a new yield function, and used aluminum alloy (A7091) uniaxial compression experiment to test the accuracy and practicability. Dias et al. [5] used the plasticity equation to analyze the parameters of the sintered porous materials after forged. He confirmed the experiment results agreeable to the theoretical analysis of the proposed model, which can be applied to the cold forging of sintered preforms. Jha et al. [6] detailed the experiments on forming characteristics and failure mechanisms from the start of spraying iron metal powder to the completion of sintering processing. In the forming properties, they discussed the size of powder particles in detail, and also classified the types of crack to the failure mechanisms.

Nguyen et al. [7] investigated the mechanical behavior of porous functionally graded (FG) beams, they indicated that it is necessary to take the effect of porosities into consideration in the vibration analysis of FG beams traversed by moving forces. Tzou et al. [8] used Taguchi Method to explore the wire rod drawing with rotating die under constant shear friction and further, they used DEFORM-3D commercial FEM software to simulate and obtain the effective stress, the effective strain, the velocity field, and the drawing force. Tzou et al. [9] carries out an FEM simulation on rotating compression forming of doublelayer Bounded clad ring by DEFORM-3D software. Effects of various compression conditions such as the clad ring height ratio, the frictional factor ratio, the rotating angular velocity etc on the compression characteristics are explored in this study.

Tzou's 2014 study [10] clearly indicates that the rotating compression forming of double-layer ring can reduce the compressive force and make the deformation uniform. The 2017's research [11] establishes a plastic mechanics analytical model based on slab method to explore the effects of rotating effect on the characteristics of rotating compression of porous cylinder. In addition, the latest study of Tzou et al. [12] also compared the various data of traditional compression forming of porous cylinder between slab method and FEM. This study used Deform-2D to explore the compression forming without rotating die. Therefore this present research uses Deform-3D to explore the rotating compression process of porous cylinders. Besides, the variations of compression characteristics with and without rotation can be investigated.

\section{FEM simulation}

In this paper, the commercial software, Deform $3 \mathrm{D}$, is used to simulate the rotating compression of porous cylinder. This software can provide the rigid, plastic, elastic-plastic and porous material simulation of the metal forming in large deformation. The FEM software Deform 3D enables to do the tool design and product design. The FEM simulations provide 3D analysis with the tetrahedron elements for meshing the work-piece of bulk forming and the auto re-mesh step is enveloped in the software package if the case 
is occurred. Using the FEM software, it can reduce the time and cost consumption of die and tool design significantly. The effective stress distribution, flow pattern, effective strain distribution, velocity field of work-piece and load stoke can be simulated by FEM software. These simulation results can be used to obtain the product geometric profile and material properties required. In the pre-process of modelling, the geometric profile of porous cylinder can be constructed easily. The porous material analysis in rotating compression process is chosen and the material properties such as Young's modulus, Poisson's ratio, are needed. For the first stage of exploring the rotating compression of porous cylinder, the isotropic work-hardening rule is assumed in flow rule due to plastic strain hardening. The green yield criterion is used. The initial conditions of work-piece are set up, and the contact along work-piece and dies are defined.

The top die with a downward speed is $1 \mathrm{~mm} / \mathrm{sec}$, and the bottom die with the angular velocity are $0,0.1,0.5,1 \mathrm{rad} / \mathrm{sec}$, all rigid contact bodies are assumed as insulation, and the heat conduction phenomenon during the porous cylinder is neglected. In the rotating compression of porous cylinder, the relationship between strain and stress is nonlinear. The DEFORM 3D has been developed to solve the nonlinear problem. The green yield criterion is available in DEFORM-3D commercial software. The contact bodies are regarded as rigid surface. The Deform pre-process are shown in Fig. 1.

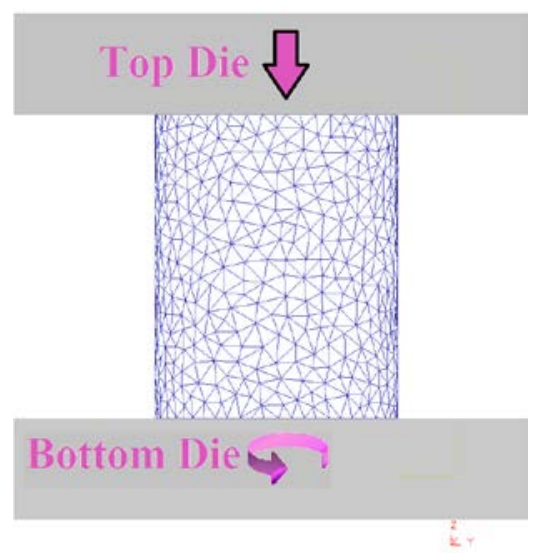

Fig. 1. The pre-process of rotating compression for porous cylinder.

\section{Results and discussions}

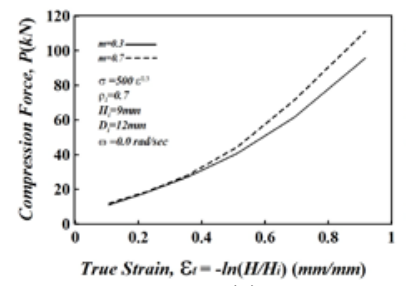

(a)

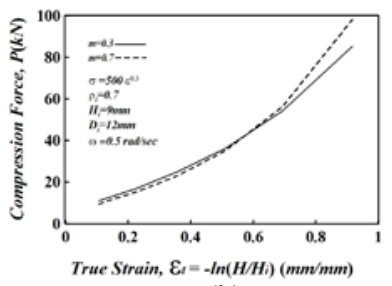

(b)

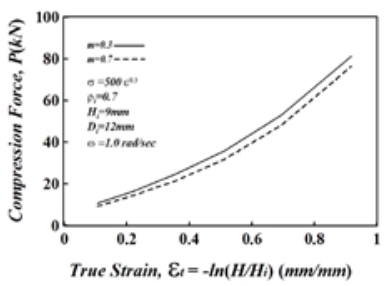

(c)

Fig. 2. Variations of the compression force with true strain for different rotating speeds $(\mathrm{Hi}=9 \mathrm{~mm}$, $\mathrm{Hi} / \mathrm{Di}=0.75$ ). 


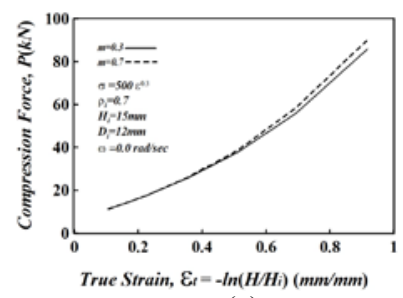

(a)

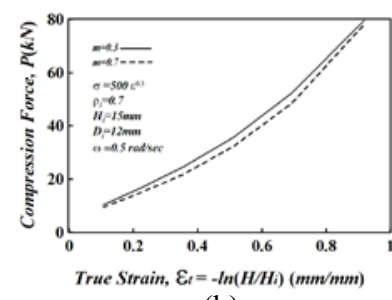

(b)

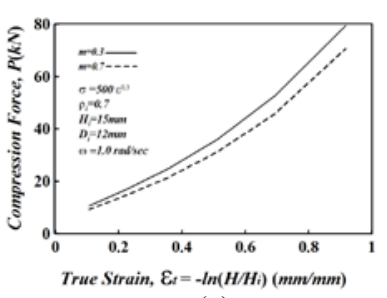

(c)

Fig. 3. Variations of the compression force with true strain for different rotating speeds ( $\mathrm{Hi}=15 \mathrm{~mm}$, $\mathrm{Hi} / \mathrm{Di}=1.25$ ).

Figure 2 shows variations of the compression force with true strain for different rotating speeds $\left(\mathrm{H}_{\mathrm{i}}=9 \mathrm{~mm}, \mathrm{H}_{\mathrm{i}} / \mathrm{D}_{\mathrm{i}}=0.75\right)$. For the compression of sound cylinder with and without rotation, as the frictional factor increases the compression force increases. However, for the porous cylinder compression, as the rotating angular speed increases gradually, whereas the compression force with higher frictional factor is smaller than that with smaller frictional factor. Figure 3 shows variations of the compression force with true strain for different rotating speeds $\left(\mathrm{H}_{\mathrm{i}}=15 \mathrm{~mm}, \mathrm{H}_{\mathrm{i}} / \mathrm{D}_{\mathrm{i}}=1.25\right)$. From this figure, the smaller compression force for higher frictional factor under $\mathrm{Hi} / \mathrm{Di}=1.25$ due to the increase of rotating angular speed is more obvious than that for $\mathrm{Hi} / \mathrm{Di}=0.75$.

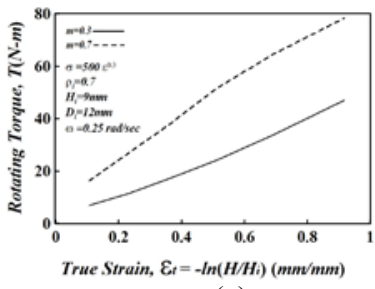

(a)

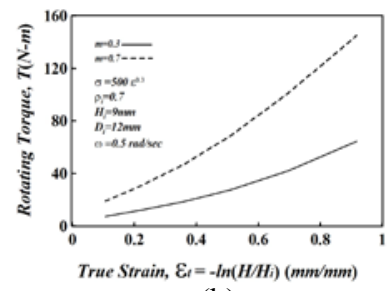

(b)

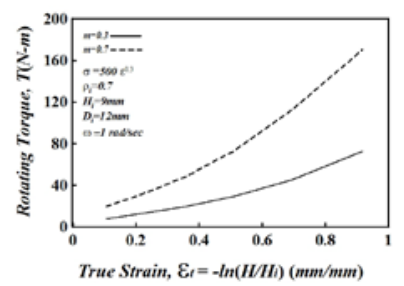

(c)

Fig. 4. Variations of the rotating torque with true strain for different rotating speeds $(\mathrm{Hi}=9 \mathrm{~mm}$, $\mathrm{Hi} / \mathrm{Di}=0.75)$

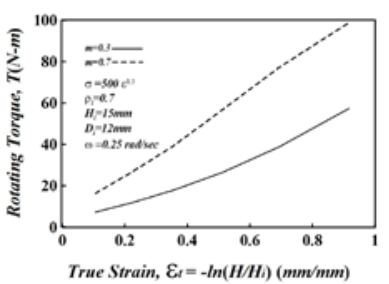

(a)

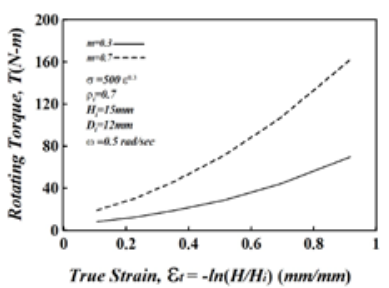

(b)

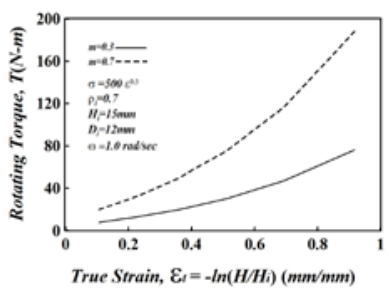

(c)

Fig. 5. Variations of the rotating torque with true strain for different rotating speeds $(\mathrm{Hi}=15 \mathrm{~mm}$, $\mathrm{Hi} / \mathrm{Di}=1.25$ ).

Figure 4 shows variations of the rotating torque with true strain for different rotating speeds $\left(\mathrm{H}_{\mathrm{i}}=9 \mathrm{~mm}, \mathrm{H}_{\mathrm{i}} / \mathrm{D}_{\mathrm{i}}=0.75\right)$. Figure 5 shows variations of the rotating torque with true strain for different rotating speeds $\left(\mathrm{H}_{\mathrm{i}}=15 \mathrm{~mm}, \mathrm{H}_{\mathrm{i}} / \mathrm{D}_{\mathrm{i}}=1.25\right)$. Regardless of sound cylinder or porous cylinder with and without rotation, as the frictional factor and rotating speed increase the rotating torque increases. Under the higher aspect ratio $\left(\mathrm{H}_{\mathrm{i}} / \mathrm{D}_{\mathrm{i}}=1.25\right)$, the rotating torque is higher. 


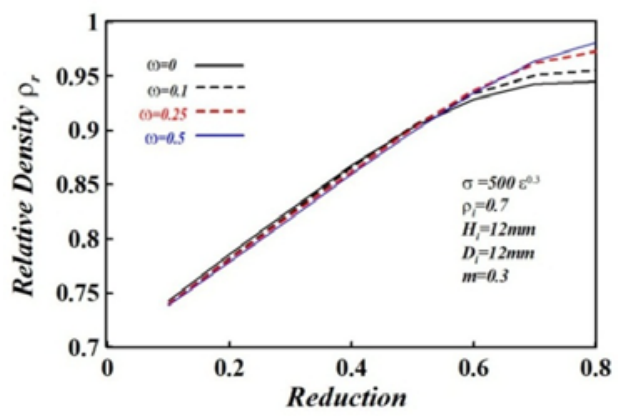

Fig. 6. The effect of different rotating speeds on relative density.

Figure 6 shows the effect of different rotating speeds on relative density. The relative density increases with the increase of reduction and rotating speed. This result can be seen in Figure 7. In Figure 7, the bulging effect can be reduced with the increase of rotating speed. And higher and uniform density can be seen under the higher rotating speed.

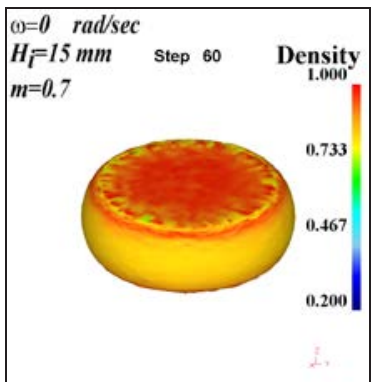

(a)

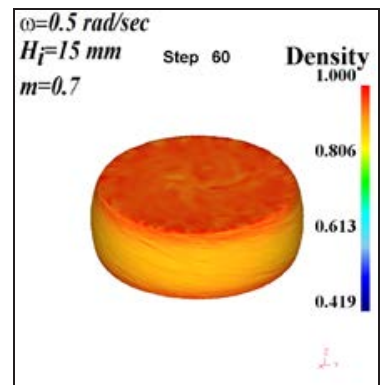

(b)

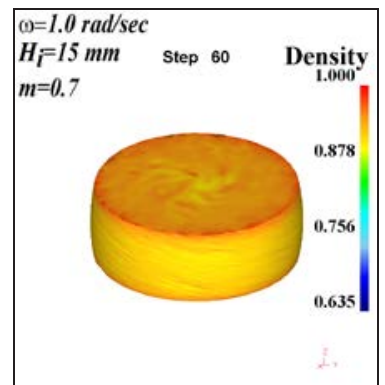

(c)

Fig. 7. Variations of density and bulging with different rotating speeds for $\mathrm{r}=60 \%$.

Figure 8 shows bulging ratio for various frictional factors. Definition of bulging ratio is that subtraction between the maximum bulging diameter and top diameter is divided by the top diameter. As the bulging ratio is increased, that indicates the bulging effect is obvious. The bulging ratio is increased with the increase of reduction and frictional factor.

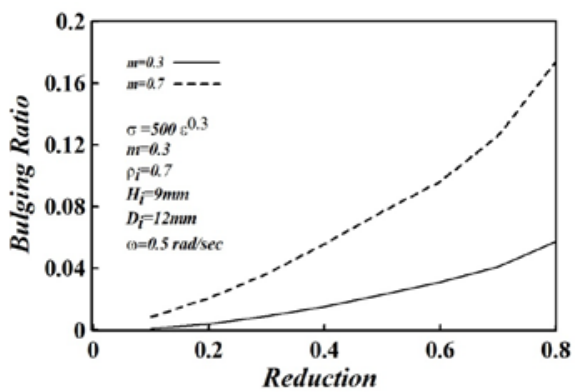

Fig. 8. Bulging ratio for various frictional factors. 


\section{Conclusions}

This study uses Deform-3D commercial software to simulate the rotating compression of porous cylinder, the major results are summarized as below:

(1)As the rotating speed increases fast, the compression force of porous cylinder is whereas reduced. This phenomenon is different from the compression of sound cylinder.

(2)The compression force under the lower aspect ratio can be reduced by the lubrication.

(3) The relative density can be increased by increasing the rotating speed and reduction ratio.

(4) The rotating torque is increased with higher rotating speed, higher frictional factor, and higher aspect ratio.

(5)The bulging effect can be reduced by increasing the rotating speed, however it is increased by increasing the frictional factor.

\section{References}

1. T. S. Yang, Y. C. Hsu, Journal of Materials Processing Technology, 177, 154-158 (2006)

2. D. N. Lee, H. S. Kim, Powder Metallurgy, 35, 275-279 (1992)

3. S. Shima, M. Oyane, International Journal of Mechanical Sciences, 18, 285-291 (1976)

4. S. M .Doraivelu, H. L. Gegel, J. S. Gunasekera, J. C. Malas, J. T. Morgan, International Journal of Mechanical Sciences, 26, 527-535 (1984)

5. A. D. M. Dias, L. Schaeer, International Journal of Machining Tools and Manufacture, 33, 577-586 (1993)

6. A. K. Jha, S. Kumar, International Journal of Machine Tool Design \& Research, 26, 369-384 (1986)

7. D.K. Nguyen, T.T. Tran, S.G. Buntara, V.T. Bui, International Journal of Engineering and Technology Innovation 6, 3, 173-189 (2016)

8. G.Y. Tzou, Y.M. Ling, S.Y. Hsia, Journal of Precision Machinery and Manufacturing Technology 5, 2, 8-16 (2015)

9. G.Y. Tzou, T.L. Chiang, C.Y. Chiu, Journal of Precision Machinery and Manufacturing Technology 2, 2, 36-46 (2012)

10. G.Y. Tzou, Y.M. Hwang, H.Y. Teng, International Journal of Precision Engineering and Manufacturing-Green Technology 1, 3, 191-199 (2014)

11. G.Y. Tzou, Y.M. Hwang, S.C. Pan, Transactions of the Canadian Society for Mechanical Engineering, 41, 571-580 (2017)

12. G.Y. Tzou, Y.M. Hwang, S.C.Pan, MATEC Web of Conferences, 123, 1-7 (2017), DOI: $10.1051 /$ matecconf/201712300002 